\title{
Early impact of Medicare Accountable Care Organizations on cancer surgery outcomes
}

Lindsey A. Herrel MD MS ${ }^{1}$, Edward C. Norton $\mathrm{PhD}^{2,3,4}$, Scott R. Hawken $\mathrm{MS}^{1}$, Zaojun Ye MS ${ }^{1}$, Brent K. Hollenbeck MD MS ${ }^{1}$, David C. Miller MD MPH ${ }^{1}$

1) Dow Division for Urological Health Services Research, Department of Urology, University of Michigan, Ann Arbor

2) Department of Health Management and Policy, University of Michigan, Ann Arbor

3) Department of Economics, University of Michigan, Ann Arbor

4) National Bureau of Economic Research

Running head: ACOs and outcomes of cancer surgery

Keywords: outcomes, Accountable Care Organizations, Medicare, surgery, health policy

Text pages: 17

Tables: 4 (plus 2 supplemental)

Figures: 1

This project was supported by funding from the National Institute of Diabetes, Digestive and Kidney Diseases (T-32-F025681 to LAH), the National Institute on Aging (R01 AG04871 to BKH) and the National Cancer Institute (1-R01-CA-174768-01-A1 to DCM).

Author Contributions

Conceptualization: LAH, ECN, SRH, ZY, DCM

Methodology: LAH, ECN, SRH, ZY, DCM

Software: LAH, ECN, ZY

Validation: LAH, ECN, ZY, DCM

Formal analysis: $\mathrm{LAH}, \mathrm{ECN}$, Investigation: LAH, ECN, ZY, DCM

Resources: LAH, ECN, ZY, DCM

Data curation: LAH, ECN, SRH, ZY, DCM

Writing (original draft): LAH, ECN, DCM

Writing (review and editing): LAH, ECN, SRH, ZY, DCM

Visualization: LAH, ECN, SRH, ZY, DCM

Supervision: LAH, ECN, DCM

Project administration: ECN, DCM

Funding acquisition: LAH, DCM

No conflicts of interest.

This is the author manuscript accepted for publication and has undergone full peer review but has not been through the copyediting, typesetting, pagination and proofreading process, which may lead to differences between this version and the Version record. Please cite this article as doi:10.1002/ cncr.30111. 
Precis: Early hospital participation in the MSSP ACO program was not associated with greater reductions in adverse perioperative outcomes for patients undergoing a major cancer surgery compared to control hospitals. The longitudinal improvements in perioperative outcomes identified during the study interval may reflect the impact of concurrent policies more directly applicable to surgical patients.

\section{Corresponding author:}

David C. Miller MD, MPH

Associate Professor

Department of Urology

University of Michigan

NCRC Building 16, Room 108E

2800 Plymouth Road

Ann Arbor, Ml 48109-2800

Phone: (734) 936-0054

Fax: (734) 232-2400

Email:demiller@med.umich.edu

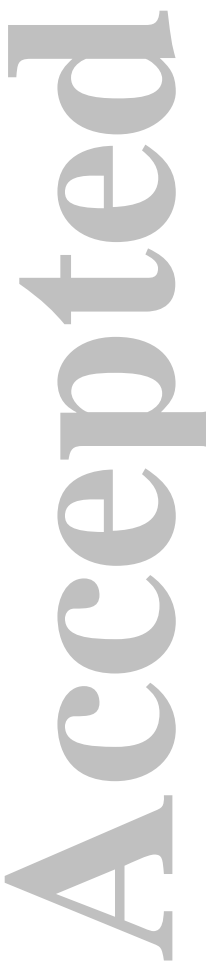




\section{Abstract}

Background: Accountable Care Organizations (ACOs) were established to improve care and outcomes for beneficiaries requiring highly coordinated, complex care. Our objective was to evaluate the association between hospital ACO participation and outcomes of major surgical oncology procedures.

Methods: We performed a retrospective cohort study of Medicare beneficiaries $>65$ years old undergoing a major surgical resection for colorectal, bladder, esophageal, kidney, liver, ovarian, pancreatic, lung or prostate cancer from 2011 through 2013. We implemented a difference-in-differences analysis comparing the post-implementation period (January 2013 through December 2013) to the baseline period (January 2011 through December 2012), to assess the impact of hospital ACO participation on 30-day mortality, complications, readmissions and length of stay.

Results: Among 384,519 patients undergoing major cancer surgery at 106 ACO hospitals and 2,561 control hospitals, we identified a 30-day mortality rate of $3.4 \%$, readmission rate of $12.5 \%$, complication rate of $43.8 \%$ and prolonged LOS rate of $10.0 \%$ in control hospitals, with similar rates in ACO hospitals. We noted secular trends, with reductions in perioperative adverse events in control hospitals between the baseline and post-implementation periods: mortality $(0.1 \%$ percentage point reduction, $p=0.19)$, readmissions $(0.4 \%, p=0.001)$, complications $(1.0 \%, p<0.001)$ and prolonged LOS $(1.1 \%, p<0.001)$. After accounting for these secular trends, we identified no significant effect of hospital participation in an ACO on the frequency of perioperative outcomes (difference-in-differences estimator p-values 0.24-0.72).

Conclusions: Early hospital participation in the MSSP ACO program was not associated with greater reductions in adverse perioperative outcomes for patients undergoing major cancer surgery compared to control hospitals. 


\section{Introduction}

Accountable care organizations (ACOs) are a signature reform of the Affordable Care Act intended to create highly integrated delivery systems that improve population health and reduce costs through increased accountability and care coordination. ${ }^{1}$ Architects of ACO policies envisioned that physicians and other healthcare workers would come together as multidisciplinary teams to coordinate and optimize care for complex and expensive patients. ${ }^{2}$ Precursors to ACOs, such as the Physician Group Practice (PGP) demonstration, provided proof-of-principle that similar models can achieve gains in the quality and cost of care provided to medical patients with multiple comorbidities. $^{3}$

Despite this evidence from primary care, it remains unknown whether ACO participation will have similar benefits for delivery of more technically complex, specialist-oriented services. Given its organization around multidisciplinary provider teams, cancer care represents an important clinical domain for evaluating this question. It is possible, for instance, that hospital ACO participation serves as a catalyst for developing integrated teams that collaborate to improve care processes and outcomes for patients undergoing cancer treatment. In this scenario, one group that may derive early benefits from the ACO model is patients undergoing major cancer surgery. For these patients with cancer, the heightened focus on quality and care coordination that accompanies ACO participation might translate quickly into improved perioperative outcomes.

In this context, we used national Medicare data to examine the early impact of hospital ACO participation on outcomes with major cancer surgery. We specifically performed a difference-in-differences analysis to examine the association between hospital participation in a Medicare Shared Savings Program (MSSP) ACO and length 
of stay, 30-day mortality, major complications, and readmissions after major oncological surgery. We hypothesize that benefits from ACO implementation would most likely occur in the form of decreased rates of readmission and complications and shorter length of stay that would result from improvements in processes of care that are at the forefront of ACO quality improvement and cost savings policies.

\section{Methods}

Data Sources

We used three datasets to perform these analyses. First, we used the Medicare Shared Savings Program (MSSP) ACO Provider-level Research Identifiable File (available from the Centers for Medicare and Medicaid Services (CMS)) to identify hospitals that formally participated in the MSSP during the first performance period from April 2012 through December 2013. Next, we used the American Hospital Association Annual Survey to evaluate hospital characteristics including region, number of beds, hospital profit status, hospital teaching status, number of operating rooms and electronic health record use. Finally, we used the $100 \%$ Medicare Provider Analysis and Review (MEDPAR) file from 2011 through 2013 to identify patient cohorts, demographic and clinical information, and the occurrence of our outcomes of interest.

\section{Identification of ACO participating and nonparticipating hospitals}

Using the MSSP ACO Provider-level dataset, we identified acute care and critical access hospitals that enrolled in an MSSP ACO during the first performance period. These hospitals are referred to as ACO hospitals throughout the manuscript; conversely, hospitals that were not formal MSSP ACO participants are referred to as 
control hospitals. Hospitals with fewer than 10 oncologic procedures overall performed during the period of interest were excluded.

\section{Identification of study population}

We used diagnosis and procedures codes from the International Classification of Diseases, Ninth Revision, Clinical Modification (ICD-9-CM) to identify patients aged 66 to 99 years that underwent major cancer surgery during the study interval for the following nine solid organ cancers: colorectal, bladder, esophageal, kidney, liver, ovarian, pancreatic, lung or prostate cancer (Supplemental Material 1). We included only those patients who had a procedure and were discharged between January 1 , 2011 and November 30, 2013 to ensure adequate follow-up for ascertainment of postoperative outcomes. We excluded patients who had two or more different oncologic procedures on the same day or $\leq 180$ days apart.

\section{Outcome measures}

We measured four post-operative outcomes: mortality, complications, prolonged length of stay (LOS) and readmissions. Mortality, complications, and readmissions were identified within 30 days of the index cancer operation. Complications were identified using established methods based on the Complication Screening Program. ${ }^{4-6}$ Using this method, we identified events including infectious, bleeding, pulmonary, renal, cardiac, neurologic, gastrointestinal and other complications occurring during the index hospitalization or within 30 days of surgery. Prolonged LOS was defined as a LOS that exceeded $90^{\text {th }}$ percentile on a procedure specific basis.

\section{Statistical analysis}


We used Student's t-test and chi-square tests where appropriate to compare characteristics of hospitals that participated in an MSSP ACO versus those that did not. We also compared demographic characteristics for patients treated in MSSP ACO hospitals versus control hospitals.

Next, we implemented a difference-in-differences analysis to evaluate the association between hospital participation in an MSSP ACO and changes in perioperative outcomes over time compared to control hospitals. ${ }^{3,7}$ To do this, we first specified whether each hospital in our sample was an ACO hospital versus a control hospital. We then specified a time variable that reflects the period before and after MSSP ACO policy implementation. ACO hospital cases were included in the postimplementation time period starting on the specific date of ACO enrollment for that hospital (April 1, 2012, July 1, 2012 or January 1, 2013). Control hospital cases were included in the post-implementation era beginning on January 1, 2013 because the majority of ACO participating hospitals entered their contracts on this date. We refer to the time period before ACO policy implementation as baseline, and the time period after as post-implementation.

We initially fit logistic regression models for each outcome across all cancer procedures combined. For each model, we included an interaction term between hospital ACO participation and time to evaluate the effect of the ACO policy. The interaction term allows the predicted outcome to differ between patients treated in ACO hospitals and non-ACO hospitals in both the pre- and post-periods. The difference-indifferences of the predicted outcomes is then the causal effect of ACO on each outcome, controlling for trends in the control group. ${ }^{8-10}$ We adjusted our regression models for the type of surgery (e.g., colectomy, prostatectomy); patient characteristics including age, gender, race, and comorbidities (using the Elixhauser method); and 
hospital characteristics including, geographic region, profit status, teaching status, rural versus urban location, and cancer procedure volume. ${ }^{11}$ For each of these models, we implemented robust standard errors to account for clustering of patient outcomes within hospitals.

In addition to the overall models, we also fit similar cancer procedure-specific models for each of the perioperative outcomes. Finally, we performed three sensitivity analyses. First, we evaluated the effect of setting the post-implementation time point for control hospital cases at July 1, 2012, rather than January 1, 2013. Second, we adjusted our overall model for three covariates that may act as markers of integration within a hospital delivery system including electronic health record use, familiarity with managed care contracts (Medicare Advantage penetration) and participation in a hospital network. Last, we evaluated outcomes only in hospital referral regions containing both an ACO hospital and a control hospital. All statistical analyses were performed using Stata version 14 (StataCorp LP, College Station, Texas); p-values $<0.05$ were considered statistically significant. The University of Michigan Institutional Review Board deemed this study exempt from review.

\section{Results}

We identified more than 380,000 patients that underwent major cancer surgery at 106 ACO hospitals and 2,561 control hospitals from 2011 through 2013. ACO hospitals were concentrated in the Northeast and Midwest regions, were more often non-profit, urban, and teaching hospitals, and had a greater number of hospital beds compared to control hospitals (Table 1).

We observed small but statistically significant differences in the populations served by these two hospital groups. For instance, patients treated at ACO hospitals 
were more often white, and had a higher prevalence of measured comorbid conditions (Table 2). Although statistically significant, differences in the mix of oncological procedures between ACO hospitals and control hospitals were small, with ACO hospitals performing more prostate and ovarian cancer surgeries, and control hospitals performing more bladder, lung and liver cancer procedures.

In the baseline study interval, unadjusted rates of 30-day mortality (3.3\% vs. $3.4 \%, p=0.54), 30$-day readmission $(12.5 \%$ vs. $12.4 \%, p=0.69)$, complications $(43.6 \%$ vs. $43,4 \% p=0.65)$, and prolonged LOS $(10.1 \%$ vs. $10.2 \% p=0.56)$ were similar between ACO hospitals and control hospitals. The adjusted rates for these events were also comparable at baseline between ACO participants and control hospitals (Table 3). We found ACO hospitals did not improve at a significantly accelerated rate compared to control hospitals. We noted secular trends, with reductions in perioperative adverse events in control hospitals between the baseline and postimplementation periods: mortality $(0.1 \%$ percentage point reduction, $\mathrm{p}=0.19)$, readmissions $(0.4 \%, p=0.001)$, complications $(1.0 \%, p<0.001)$ and prolonged LOS $(1.1 \%, p<0.001)$. After accounting for these secular trends, we identified no significant effect of hospital participation in an ACO on the frequency of any perioperative outcomes ( $p$-values for difference-in-differences estimator 0.24-0.72, Figure 1).

Finally, when we examined the association between hospital ACO participation and perioperative outcomes for individual cancers we noted similar patterns, with no greater improvements in perioperative outcomes for ACO hospitals (Supplemental Material 2). Table 4 presents site-specific outcomes for several cancers comparing post-implementation outcomes to baseline. We noted no substantive changes in our results in sensitivity analyses where the post-implementation date for control hospitals was changed from January 1, 2013 to July 1, 2012, when controlling for additional 
measures of health system integration or when limiting our analyses to only markets containing both an ACO hospital and a control hospital.

\section{Discussion}

Our study has two principal findings. First, early hospital participation in the MSSP ACO program did not accelerate improvements in several adverse events (i.e., 30-day mortality, readmissions, major complications, and prolonged LOS) after major cancer surgery compared to control hospitals. Second, with the exception of mortality, rates of these adverse perioperative outcomes are improving across hospitals over time, with $0.1-1.6 \%$ percentage point reductions in the frequency of observed adverse events from baseline to post-implementation of ACO policies.

Our findings showing no greater benefits with mortality or length of stay for patients undergoing cancer surgery in ACO hospitals is consistent with prior work evaluating outcomes for beneficiaries with cancer during the Physician Group Practice (PGP) demonstration project. ${ }^{12}$ Namely, cancer patients treated at facilities participating in PGP had rates of in-hospital mortality that were equivalent to those for a similar patient cohort treated in non-PGP hospitals. Likewise, there was no effect of PGP participation on the number of days that patients with cancer spent in the hospital.

Although not attributable to ACO participation, there are several potential explanations for the observed decline in readmissions and complications over the study interval. For instance, work by others has demonstrated similar trends associated with concurrent CMS pay-for-performance initiatives including the Hospital Readmission Reduction Program (HRRP), Value Based Purchasing (VBP) program, and the HospitalAcquired Conditions Reduction program (HAC). ${ }^{13-16}$ While not directed specifically at patients undergoing cancer surgery, processes developed to reduce readmissions in 
response to the HRRP, particularly those directed at surgical admissions, may well have spillover benefits for other surgical patients. Likewise, improvements in care processes and patient safety in response to VBP and HAC metrics may have the collateral benefit of shortening length of stay and reducing readmissions for patients undergoing major cancer surgery. ${ }^{17}$ Accordingly, the observed longitudinal improvements in cancer surgical outcomes are likely related to a combination of initiatives implemented during the same time period as the MSSP ACO program.

Our study has several limitations. First, most ACO quality metrics are not specifically focused on improving cancer care. Accordingly, further improvements in care delivery may require policies directed at cancer care specifically, rather than more general initiatives like ACOs. However, while these specific surgical outcome measures may not map directly to MSSP ACO quality measures, improvements in perioperative outcomes should translate into lower costs of care, which is highly relevant for ACO performance. It is also conceivable that incremental improvements in cancer care may be more difficult to achieve through the ACO model since there has long been a focus on care coordination and quality measurement in this patient population. Moreover, organizational change may require more than one or two years and effects derived from ACO policies may become stronger over time and as more organizations form. Second, we did not evaluate for improvements in cancer screening, surveillance and end of life care. In many ways, these domains of cancer care may be more responsive to the ACO model than surgical outcomes, from both a cost and quality perspective. As a result, future evaluations of cancer care and outcomes in ACOs are needed to define the impact of this model on these distinct phases of cancer care ${ }^{18}$ Third, our study assumes there were no inherent differences between control hospitals and hospitals that ultimately joined an ACO. While, ACO hospitals and control 
hospitals are not identical, they have similar patient populations and had equivalent outcome rates at baseline. Additionally, our study design assumes that outcome trends during the baseline period were similar among ACO hospitals and control hospitals. As more data becomes available, evaluating time trends across the baseline and postimplementation periods will be important to consider. Finally, hospital affiliation with an ACO represents one mechanism for ACO participation. Additionally, physicians can align with an ACO independent of hospital participation. While our study does not examine the role of physician ACO participation, control hospitals with a large number of ACO participating physicians may be influenced by ACO policies.

These limitations notwithstanding our findings have important implications for ACO leadership and policymakers. For ACO leadership, simply committing to the framework, measures, and payment changes that come with ACO participation will not necessarily translate into short-term improvements in perioperative outcomes for cancer patients. Nonetheless, our findings showing improvements in care regardless of hospital ACO status suggests that ACOs may benefit from other ongoing quality improvement programs that are impacting care at hospitals nationwide. For instance, reducing surgical site infections after colectomy will positively impact hospital performance with both the VBP and HAC programs, while also reducing costs for the ACO.

For policymakers, our findings suggest that, at least in this early period, innovative policies based on ACO principles (e.g., primary care focus) may have limited impact on inpatient surgical care. Programs directed specifically at improving surgical and cancer specific outcomes—such as surgical quality improvement collaboratives or initiatives through oncology groups such as the American Society of Clinical Oncology's Institute for Quality — may offer alternative and more direct ways for physicians, patients 
and health systems to partner to improve outcomes and reduce costs with major cancer surgery. ${ }^{19,20}$

Although longer follow-up is needed, early hospital participation in the MSSP ACO program was not associated with greater reductions in adverse perioperative outcomes for patients undergoing a major cancer surgery compared to nonparticipating hospitals. The longitudinal improvements in perioperative outcomes identified during the study interval may reflect the impact of concurrent policies more directly applicable to surgical patients. Moving forward, studies that inform the impact of ACOs at other points in the cancer care continuum (e.g., early detection, end-of-life care) will further clarify the relevance and impact of this model in oncology.

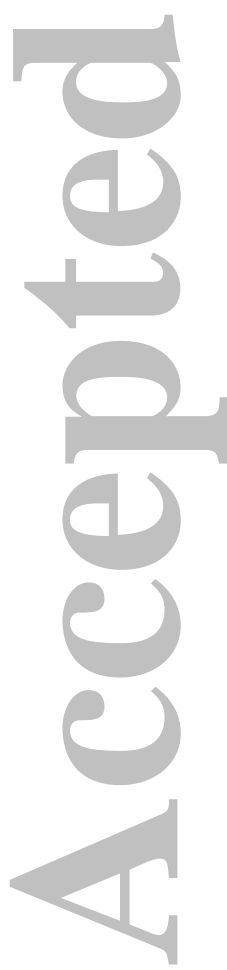




\section{Acknowledgements}

This project was supported by funding from the National Institute of Diabetes, Digestive and Kidney Diseases (T-32-F025681 to LAH), the National Institute on Aging (R01 AG04871 to BKH) and the National Cancer Institute (1-R01-CA-174768-01-A1 to DCM).

No funding organization or sponsor had a role in the design and conduct of the study; management, analysis, or interpretation of the data; preparation, review or approval of the manuscript; or decision to submit the manuscript for publication.

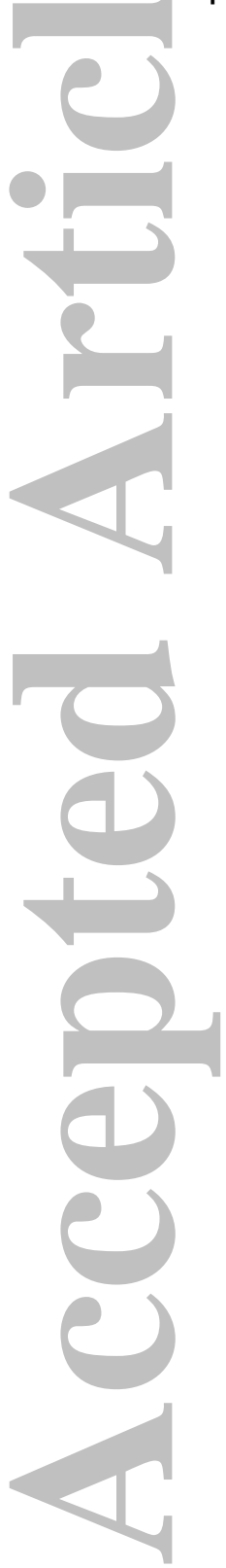




\section{References:}

1. Berwick DM, Nolan TW, Whittington J. The triple aim: Care, health, and cost. Health Aff. 2008;27(3):759-769.

2. Berwick DM. ACOs--promise, not panacea. JAMA. 2012;308(10):1038-1039.

3. Colla $\mathrm{CH}$, Wennberg DE, Meara E, et al. Spending differences associated with the Medicare Physician Group Practice Demonstration. JAMA.

2012;308(10):1015-1023.

4. Jezzoni LI, Mackiernan YD, Cahalane MJ, Phillips RS, Davis RB, Miller K.

Screening inpatient quality using post-discharge events. Med Care. 1999;37(4):384-398.

5. Osborne NH, Nicholas L, Ryan AM, Thumma JR, Dimick JB. Association of Hospital Participation in a Quality Reporting Program With Surgical Outcomes and Expenditures for Medicare Beneficiaries. JAMA. 2015;313(5):496-504.

6. Tan H-J, Norton EC, Ye Z, Hafez KS, Gore JL, Miller DC. Long-term survival following partial vs radical nephrectomy among older patients with early-stage kidney cancer. JAMA. 2012;307(15):1629-1635.

7. Dimick JB, Ryan AM. Methods for evaluating changes in health care policy: The difference-in-differences approach. JAMA. 2014;312(22):2401-2402.

8. Norton EC. Computing interaction effects and standard errors in logit and probit models. Stata J. 2004;4(2):154-167.

9. Karaca-Mandic P, Norton EC, Dowd B. Interaction Terms in Nonlinear Models. Health Serv Res. 2012;47(1pt1):255-274.

10. Ai C, Norton EC. Interaction terms in logit and probit models. Econ Lett. 2003;80(1):123-129.

11. Elixhauser A, Steiner C, Harris DR, Coffey RM. Comorbidity measures for use 
with administrative data. Med Care. 1998;36(1):8-27.

2. Colla $\mathrm{CH}$, Lewis VA, Gottlieb DJ, Fisher ES. Cancer spending and accountable care organizations: Evidence from the Physician Group Practice Demonstration. Healthc (Amsterdam, Netherlands). 2013;1(3-4):100-107.

3. Zuckerman RB, Sheingold SH, Orav EJ, Ruhter J, Epstein AM. Readmissions, Observation, and the Hospital Readmissions Reduction Program. N Engl J Med. 2016:NEJMsa1513024.

4. Kahn CN, Ault T, Potetz L, Walke T, Chambers JH, Burch S. Assessing Medicare's hospital pay-for-performance programs and whether they are achieving their goals. Health Aff (Millwood). 2015;34(8):1281-1288.

5. Gerhardt G, Yemane A, Hickman P, Oelschlaeger A, Rollins E, Brennan N. Medicare readmission rates showed meaningful decline in 2012. Medicare Medicaid Res Rev. 2013;3(2).

6. Centers for Medicare \& Medicaid Services. Medicare Hospital Quality Chartbook. 2012. http://cms.gov/Medicare/Quality-Initiatives-Patient-AssessmentInstruments/HospitalQualitylnits/Downloads/MedicareHospitalQualityChartbook20 12.pdf.

7. Rajaram R, Chung JW, Kinnier C V, et al. Hospital Characteristics Associated With Penalties in the Centers for Medicare \& Medicaid Services Hospital-Acquired Condition Reduction Program. JAMA. 2015;314(4):375-383.

8. Carlin CS, Dowd B, Feldman R. Changes in Quality of Health Care Delivery after Vertical Integration. Health Serv Res. 2014:1-26.

9. Healy MA, Krell RW, Abdelsattar ZM, et al. Pancreatic Resection Results in a Statewide Surgical Collaborative. Ann Surg Oncol. 2015:12-16.

0. Waits SA, Fritze D, Banerjee M, et al. Developing an argument for bundled 
interventions to reduce surgical site infection in colorectal surgery. Surgery. 2014;155(4):602-606.
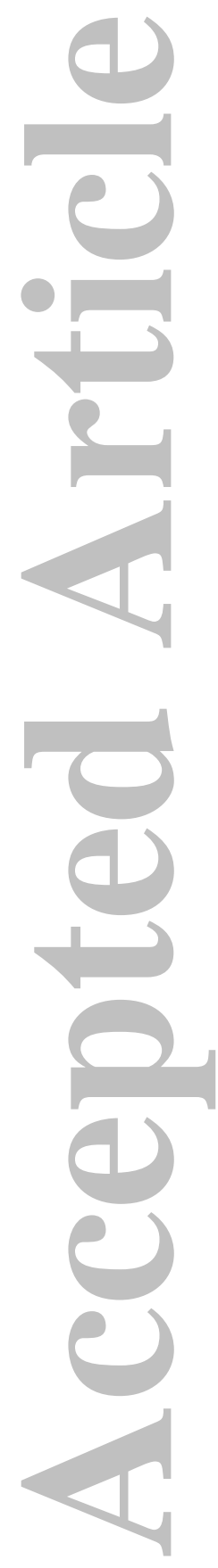
Figure Legend.

Figure 1. Change in rates of perioperative outcomes after ACO implementation.

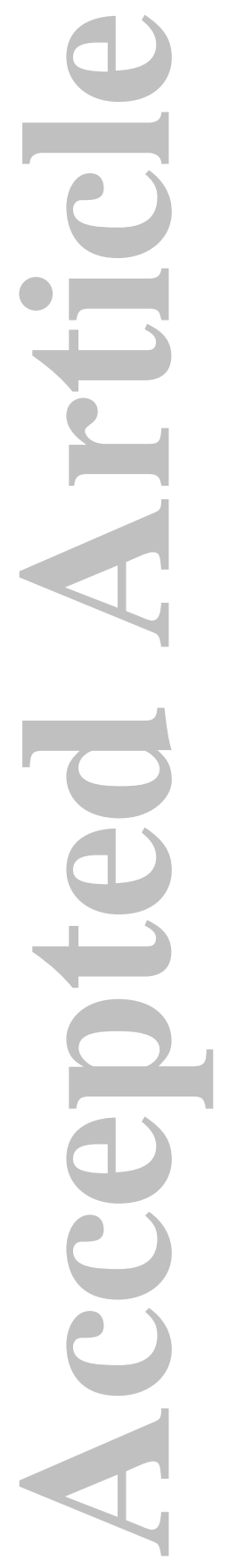




\section{Tables}

Table 1. Characteristics of ACO hospitals versus control hospitals

\begin{tabular}{|c|c|c|c|}
\hline Mean (SD) & $\begin{array}{r}\text { ACO hospitals } \\
(n=106)\end{array}$ & $\begin{array}{r}\text { Control hospitals } \\
(n=2,561)\end{array}$ & p-value \\
\hline Annual oncologic surgical volume, median (IQR) & $31.3(10.7-90.3)$ & $23.3(9-58.7)$ & 0.06 \\
\hline Geographic region (\%) & & & $<0.001$ \\
\hline Northeast & 25.5 & 17.1 & \\
\hline Midwest & 48.1 & 25.0 & \\
\hline South & 23.6 & 37.9 & \\
\hline West & 2.8 & 20.2 & \\
\hline Number of beds & & & 0.02 \\
\hline$<200$ & 42.5 & 52.5 & \\
\hline $200-349$ & 24.5 & 27.0 & \\
\hline $350-499$ & 19.8 & 11.3 & \\
\hline$\geq 500$ & 13.2 & 9.3 & \\
\hline Hospital profit status (\%) & & & $<0.001$ \\
\hline For-profit & 4.7 & 17.6 & \\
\hline Non-profit & 86.8 & 69.0 & \\
\hline Public & 8.5 & 13.4 & \\
\hline \multicolumn{4}{|l|}{ Other characteristics } \\
\hline Teaching hospital (\%) & 48.1 & 34.1 & 0.002 \\
\hline Urban location (\%) & 84.0 & 72.4 & 0.01 \\
\hline Number of operating rooms, median (IQR) & $11(5-19)$ & $9(5-14)$ & 0.09 \\
\hline Electronic health record implemented (\%) & 95.7 & 98.1 & 0.24 \\
\hline Medicare Advantage penetration (\%) ${ }^{*}$ & 25.9 & 25.7 & 0.90 \\
\hline Network participant & $51.4 \%$ & $40.7 \%$ & 0.03 \\
\hline
\end{tabular}

*Medicare Advantage penetration is reported at the county level 
Table 2. Characteristics of beneficiaries treated at ACO hospitals versus control hospitals

\begin{tabular}{|c|c|c|c|c|c|c|}
\hline \multirow[b]{2}{*}{ Mean } & \multicolumn{2}{|c|}{ Baseline } & \multicolumn{2}{|c|}{ Post-implementation } & \multirow[b]{2}{*}{$\begin{array}{l}\text { Differential change } \\
\text { for ACO versus } \\
\text { control hospitals }\end{array}$} & \multirow[b]{2}{*}{$\mathrm{p}$-value } \\
\hline & $\begin{array}{c}\text { ACO } \\
\text { hospitals } \\
(n=10,347) \\
\end{array}$ & $\begin{array}{c}\text { Control } \\
\text { hospitals } \\
(n=252,627)\end{array}$ & $\begin{array}{c}\text { ACO } \\
\text { hospitals } \\
(n=9,092) \\
\end{array}$ & $\begin{array}{c}\text { Control } \\
\text { hospitals } \\
(n=112,453) \\
\end{array}$ & & \\
\hline Age & 74.44 & 74.49 & 74.15 & 74.39 & -0.20 & 0.002 \\
\hline Female (\%) & 42.27 & 41.87 & 42.19 & 42.11 & -0.32 & 0.42 \\
\hline Race (\%) & & & & & & $<0.001$ \\
\hline White & 87.63 & 86.58 & 87.25 & 86.04 & 0.16 & \\
\hline Black & 8.74 & 8.17 & 8.69 & 8.14 & -0.02 & \\
\hline Other & 3.63 & 5.25 & 4.06 & 5.83 & -0.15 & \\
\hline \multicolumn{7}{|l|}{ Cancer Surgery (\%) } \\
\hline Bladder & 4.09 & 4.27 & 3.86 & 4.45 & -0.41 & 0.02 \\
\hline Prostate & 18.37 & 17.40 & 17.21 & 16.21 & 0.03 & 0.004 \\
\hline Esophageal & 0.98 & 1.12 & 1.12 & 1.20 & 0.06 & 0.19 \\
\hline Pancreas & 2.95 & 3.04 & 3.28 & 3.21 & 0.16 & 0.93 \\
\hline Lung & 17.19 & 17.55 & 17.69 & 18.21 & -0.16 & 0.24 \\
\hline Liver & 0.74 & 1.04 & 1.03 & 1.16 & 0.17 & 0.01 \\
\hline Kidney & 11.82 & 12.11 & 12.23 & 12.69 & -0.17 & 0.25 \\
\hline Colorectal & 39.03 & 39.00 & 38.35 & 38.26 & 0.06 & 0.86 \\
\hline Ovarian & 4.83 & 4.47 & 5.22 & 4.60 & 0.26 & 0.001 \\
\hline \multicolumn{7}{|l|}{ Comorbid diseases (\%) } \\
\hline Congestive heart failure & 8.80 & 7.74 & 8.36 & 7.39 & -0.09 & $<0.001$ \\
\hline Valvular disease & 5.99 & 5.77 & 5.55 & 5.89 & -0.56 & 0.91 \\
\hline Pulmonary hypertension & 2.37 & 1.94 & 2.42 & 1.96 & 0.04 & $<0.001$ \\
\hline Peripheral vascular disease & 7.40 & 6.89 & 6.74 & 7.25 & -1.02 & 0.62 \\
\hline Paralysis & 1.10 & 0.93 & 0.87 & 0.90 & -0.20 & 0.33 \\
\hline Other neurological disorders & 3.63 & 3.17 & 3.10 & 3.24 & -0.59 & 0.14 \\
\hline Chronic pulmonary disease & 24.81 & 22.92 & 23.90 & 22.92 & -0.90 & $<0.001$ \\
\hline Diabetes w/o complication & 23.54 & 22.45 & 24.42 & 22.77 & 0.56 & $<0.001$ \\
\hline Diabetes with complication & 2.50 & 2.72 & 3.32 & 2.94 & 0.60 & 0.43 \\
\hline Hypothyroidism & 11.48 & 12.21 & 12.65 & 13.02 & 0.35 & 0.08 \\
\hline Renal failure & 0.80 & 0.84 & 0.83 & 0.87 & 0.00 & 0.56 \\
\hline Liver disease & 0.74 & 1.04 & 1.03 & 1.16 & 0.17 & 0.01 \\
\hline Lymphoma & 0.67 & 0.74 & 0.70 & 0.75 & 0.03 & 0.38 \\
\hline $\begin{array}{l}\text { Metastatic cancer } \\
\text { Solid tumor without mets }\end{array}$ & 18.14 & 19.06 & 18.98 & 18.48 & 1.43 & 0.23 \\
\hline (other than primary) & 9.43 & 9.53 & 9.76 & 9.65 & 0.20 & 0.94 \\
\hline Rhematologic disorder & 2.27 & 2.27 & 2.62 & 2.46 & 0.16 & 0.34 \\
\hline Coagulopathy & 3.73 & 3.48 & 4.26 & 3.67 & 0.34 & 0.001 \\
\hline Obese & 9.67 & 8.91 & 10.67 & 10.33 & -0.42 & $<0.001$ \\
\hline Weight loss & 9.23 & 8.72 & 9.56 & 8.69 & 0.37 & 0.001 \\
\hline Electrolyte disorders & 22.54 & 21.86 & 23.44 & 22.18 & 0.58 & 0.001 \\
\hline Blood loss anemia & 3.88 & 3.51 & 3.56 & 3.19 & 0.01 & 0.02 \\
\hline Deficiency anemias & 21.45 & 20.92 & 21.01 & 20.12 & 0.36 & 0.06 \\
\hline Psychoses & 1.96 & 1.87 & 1.88 & 1.96 & -0.17 & 0.79 \\
\hline
\end{tabular}


Depression

Hypertension
7.95

66.17
7.43

65.29
8.69

66.97
7.95

65.65
0.21

0.44
$<0.001$

0.001

*gastrointestinal bleed, AIDS, alcohol use, drug use excluded due to small numbers or redacted data

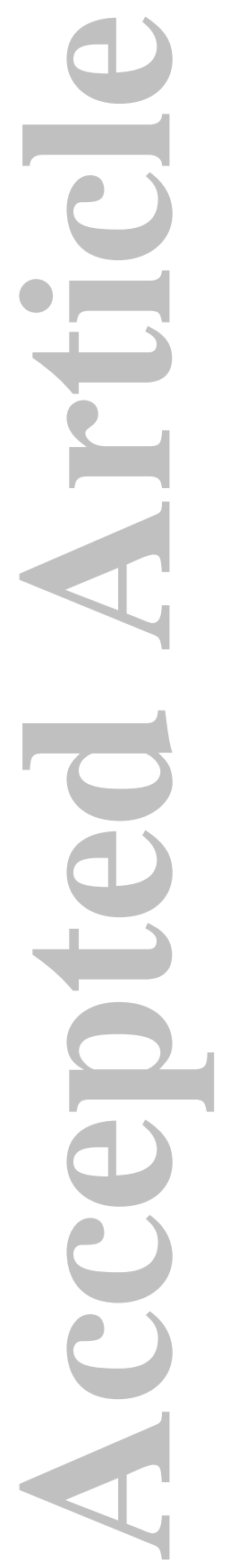


Table 3. Adjusted rates of adverse perioperative outcomes for patients undergoing cancer surgery at hospitals before and after ACO policy implementation.

\begin{tabular}{lcccc}
\hline & \multicolumn{2}{c}{ Control hospitals } & \multicolumn{2}{c}{ ACO hospitals } \\
\cline { 2 - 5 } 30-day Mortality & Baseline & Post-implementation & Baseline & Post-implementation \\
\cline { 2 - 5 } Readmissions & $3.4 \%$ & $3.3 \%$ & $3.4 \%$ & $3.2 \%$ \\
Complications & $12.5 \%$ & $12.1 \%{ }^{*}$ & $12.6 \%$ & $12.0 \%$ \\
Prolonged LOS & $43.8 \%$ & $42.7 \%{ }^{*}$ & $44.0 \%$ & $42.5 \%$ \\
\hline Adjus & $10.0 \%$ & $8.9 \% *$ & $10.0 \%$ & $8.4 \%$ \\
\hline
\end{tabular}

Adjusted for surgery type, age, gender, race, region, bed size, hospital profit status, teaching status, rural/urban location, cancer procedure volume and comorbidities

${ }^{*}$ Change from baseline $(p \leq 0.001)$

Legend: Statistically significant decline in readmissions, complications and prolonged LOS for patients undergoing a major cancer surgery at control hospitals with parallel trends in perioperative outcomes at ACO hospitals.

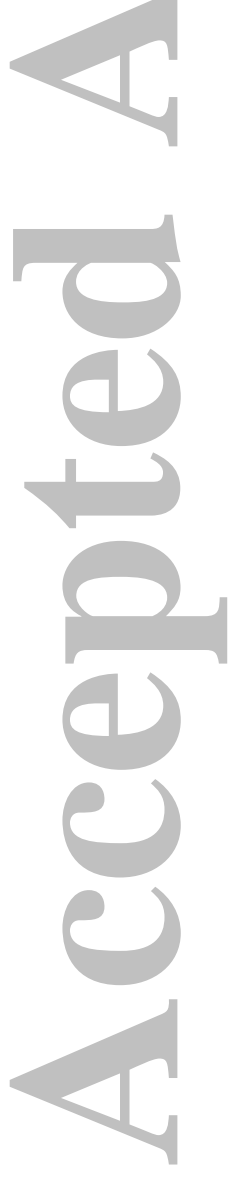


Table 4. Relative risk of adverse perioperative outcomes in control hospitals during the post-implementation versus baseline time periods $\left({ }^{*} p<0.05\right)$.

\begin{tabular}{lllll} 
& Mortality & Readmissions & Complications & Prolonged LOS \\
\cline { 2 - 5 } Prostate & $0.84(0.57-1.24)$ & $1.03(0.95-1.11)$ & $0.97(0.93-1.02)$ & $0.89(0.83-0.94)^{*}$ \\
Bladder & $1.14(0.93-1.41)$ & $1.01(0.95-1.07)$ & $0.98(0.96-1.00)$ & $0.89(0.79-1.00)$ \\
Esophagus & $0.83(0.55-1.24)$ & $1.06(0.90-1.24)$ & $0.92(0.86-0.99)^{*}$ & $0.73(0.55-0.97)^{*}$ \\
Pancreas & $0.95(0.75-1.20)$ & $1.01(0.93-1.10)$ & $0.95(0.91-0.99)^{*}$ & $0.86(0.74-0.99)^{*}$ \\
Lung & $0.98(0.89-1.07)$ & $0.91(0.87-0.95)^{*}$ & $0.92(0.90-0.95)^{*}$ & $0.88(0.84-0.92)^{*}$ \\
Liver & $0.86(0.59-1.26)$ & $0.92(0.76-1.11)$ & $0.96(0.88-1.04)$ & $0.74(0.60-0.92)^{*}$ \\
Kidney & $1.11(0.98-1.30)$ & $0.97(0.91-1.03)$ & $0.99(0.97-1.01)$ & $0.92(0.87-0.98)^{*}$ \\
Colorectal & $1.00(0.95-1.04)$ & $0.96(0.94-0.99)^{*}$ & $0.99(0.98-1.00)$ & $0.89(0.86-0.92)^{*}$ \\
Ovary & $0.71(0.59-0.86)$ & $0.98(0.90-1.06)$ & $0.98(0.95-1.01)$ & $0.92(0.84-1.02)$ \\
All cancers & $0.97(0.94-1.01)$ & $0.97(0.95-0.99)^{*}$ & $0.98(0.97-0.98)^{*}$ & $0.88(0.87-0.90)^{*}$
\end{tabular}



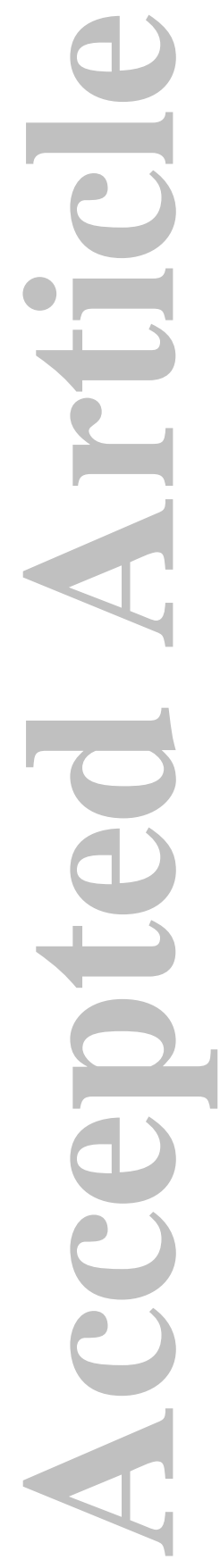

This article is protected by copyright. All rights reserved. 


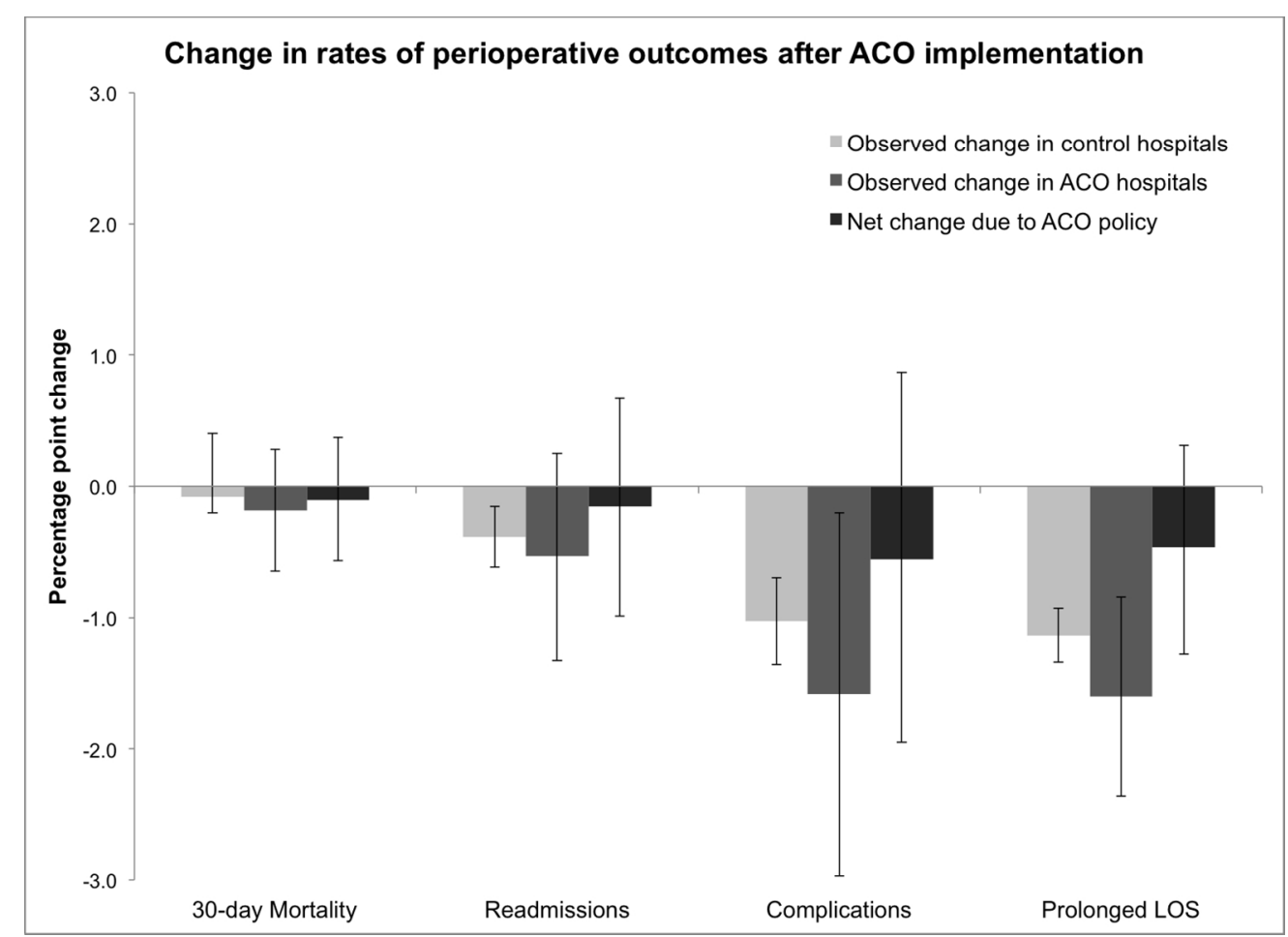

Change in rates of perioperative outcomes after ACO implementation. $268 \times 195 \mathrm{~mm}(150 \times 150 \mathrm{DPI})$ 
Supplemental Material 1. Cohort selection.

\begin{tabular}{|c|c|c|}
\hline Cancer type & Diagnosis codes (ICD-9) & Procedure codes (ICD-9) \\
\hline Pros & 185 & 603604605606160626069 \\
\hline Bladder & $\begin{array}{l}1881880188118821883 \\
18841885188618871888 \\
1889\end{array}$ & 57657757715779 \\
\hline Esophagus & $\begin{array}{l}1501500150115021503 \\
1504150515081509\end{array}$ & 4244240424142424399 \\
\hline Pancreas & $\begin{array}{l}1571570157115721573 \\
15741575157615771578 \\
1579\end{array}$ & 52525251525352552652752595222 \\
\hline Lur & $\begin{array}{l}1621620162116221623 \\
16241625162616271628 \\
1629\end{array}$ & $\begin{array}{l}322322032932293230323932432413249 \\
32503529325\end{array}$ \\
\hline Liver & 155155015511552 & 502250350450595025029 \\
\hline Kidney & $\begin{array}{l}189189018900189011898 \\
189123691\end{array}$ & 55455515552555455345532555 \\
\hline Color & $\begin{array}{l}15301531153215331534 \\
15351536153715381539 \\
15401541\end{array}$ & $\begin{array}{l}4574571457245734574457545764579 \\
4849458145824583458486148654869 \\
48624863486448504851485248594840 \\
484148424843485486484173217331734 \\
173517361739\end{array}$ \\
\hline Oval & 1830 & $\begin{array}{l}6526531653965516552655365546541 \\
65496561656265636564664665654656 \\
68868368316839684684168496856851 \\
68596866861686968768716879689541 \\
54115421544\end{array}$ \\
\hline
\end{tabular}

This article is protected by copyright. All rights reserved. 
Supplemental Material 2. Percentage point change in adverse perioperative outcomes attributable to ACO policy (difference-in-differences estimator) by cancer type $\left({ }^{*} p<0.05\right.$ )

\begin{tabular}{lllll} 
& Mortality & Readmissions & Complications & Prolonged Los \\
\hline Prostate & $0.01(-0.21-0.22)$ & $-1.44(-2.93-0.08)$ & $0.43(-1.75-2.61)$ & $0.38(-0.94-1.63)$ \\
Bladder & $-1.59(-4.75-1.50)$ & $-3.51(-9.61-2.48)$ & $-4.15(-10.05-2.31)$ & $-0.03(-4.23-3.96)$ \\
Esophagus & $2.19(-2.13-7.67)$ & $-8.67(-21.88-2.52)$ & $-10.19(-26.33-5.30)$ & $2.73(-8.12-13.63)$ \\
Pancreas & $2.39(-0.68-5.43)$ & $3.70(-2.10-9.23)$ & $-3.92(-12.43-4.39)$ & $-0.28(-3.41-3.15)$ \\
Lung & $-0.16(-1.21-0.87)$ & $1.21(-1.14-3.57)$ & $-0.22(-3.63-3.09)$ & $0.40(-1.61-2.31)$ \\
Liver & $-2.06(-4.37-1.10)$ & $3.80(-10.98-18.78)$ & $-13.3(-25.69--3.24)^{*}$ & $0.05(-19.12-19.30)$ \\
Kidney & $-0.19(-1.28-0.90)$ & $0.98(-1.20-3.16)$ & $-2.17(-6.07-1.76)$ & $-0.56(-3.08-1.95)$ \\
Colorectal & $-0.08(-1.08-0.92)$ & $-0.32(-1.97-1.30)$ & $0.45(-1.93-2.84)$ & $-0.90(-2.30-0.49)$ \\
Ovary & $0.25(-2.30-2.76)$ & $0.87(-4.18-6.53)$ & $-1.63(-8.83-5.56)$ & $-0.33(-3.35-2.74)$ \\
\hline
\end{tabular}

Legend: Negative values indicate further decline in adverse events attributable to ACO policies (benefit beyond change seen in control hospitals). 